\title{
MODELLING MICROSTRUCTURAL TRANSFORMATIONS OF NICKEL BASE SUPERALLOY IN 718 DURING HOT DEFORMATION
}

\author{
Robert P. Guest ${ }^{1}$, Sammy Tin ${ }^{2}$ \\ ${ }^{1}$ Firth Rixson Ltd, Firth House, P. O. Box 644, Meadowhall Road. Sheffield. S9 1JD \\ ${ }^{2}$ Department of Materials Science and Metallurgy, New Museum Site, \\ Cambridge University, Pembroke Street, Cambridge. CB2 3QZ
}

Keywords: Hot deformation, Recrystallisation, Microstructural evolution

\begin{abstract}
A new Inconel 718 microstructural evolution model that works in tandem with finite element software is described, and is then used to predict the microstructure during the production of a typical two-stage forging route. The model works by grouping similar grains into grainsets and predicting the dislocation density within these grainsets during thermomechanical processing. The model is shown to accurately predict both the grain size and presence of $\delta$ precipitates during the processing route, and displays results both numerically and graphically.
\end{abstract}

\section{Introduction}

Although a number of models claiming to predict the microstructural evolution of IN 718 exist (e.g. [1-12]), they either fall into the category of being too simplistic and producing only an average grain size and approximate volume fraction recrystallised, or are far too complicated and too computationaly intensive to be used on a daily basis by process engineers. A model is required, therefore, that can be run within a commercially available finite element package, or in tandem with one, that will deliver detailed results, run in a reasonable time, and be simple to use.

\section{Model Development}

It has been previously stated [13] that dynamic recrystallisation is caused by the generation of subgrains, and that these subgrains dictate the size of the recrystallised grains. Therefore, the reason given for a decreasing dynamically recrystallised grain size with increasing strain rate and decreasing temperature is that these conditions produce a higher dislocation density and a higher number of subgrains. There is, however, another explanation. For a given strain, a low strain rate or high temperature will result in more grain boundary migration. If the temperature is high, boundary migration will be faster due to kinetic reasons. If the strain rate is low, for a given strain the deformation will take longer, giving the boundaries more time to migrate than during deformation at a higher strain rate. For a high strain rate or low temperature, the opposite is true; the short time period deformation is occurring (high $\dot{\varepsilon}$ ), and the low temperature results in a small grain size. 
Figure 1 shows a series of schematic diagrams representing how the recrystallised microstructure will develop during deformation. The colour of the grains in the diagrams represents the dislocation density within the grain, with darker colours indicating a higher dislocation density. Figure 1 (a) shows the undeformed microstructure, consisting of unrecrystallised grains with a uniform low dislocation density. As the strain increases within the system, the dislocation density within the grains will also increase. This is shown in Figure 1 (b), where the dislocation density has not reached the critical dislocation density for recrystallisation. As the strain and, therefore, the dislocation density increases, the critical dislocation density required to initiate recrystallisation is reached. At this point (Figure 1 (c)), new grains with a low dislocation density can nucleate on grain boundaries within the system. As the strain continues to increase, the new grains will grow into the matrix, driven by the dislocation density difference between themselves and the matrix. As the new recrystallising grains grow, the average dislocation density within them will tend to decrease as new area is swept out by the moving boundaries, but will also increase due to the new dislocations added to the system as a result of the ongoing deformation. The process of nucleation on the original grain boundaries and growth of new grains will continue (Figures 1 (d) and (e)) until a set of recrystallising grains stops growing as its dislocation density reaches that of the matrix. At this point, the length of grain boundary available to nucleate on increases dramatically, and more nuclei form (Figure 1 (f)).

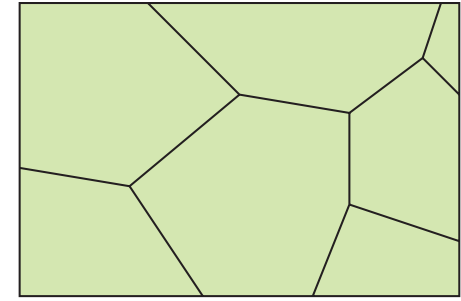

(a) Stage 1

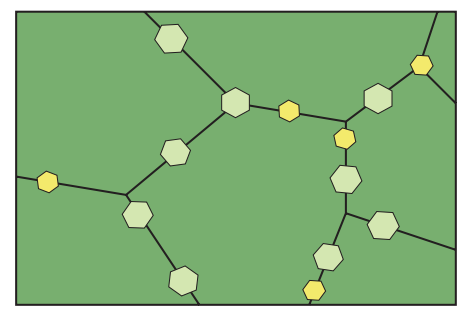

(d) Stage 4

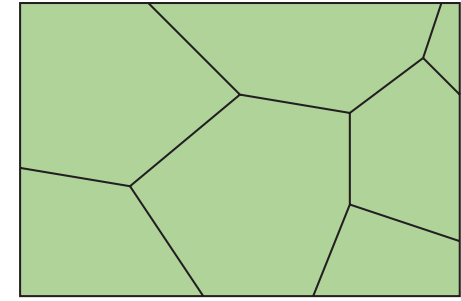

(b) Stage 2

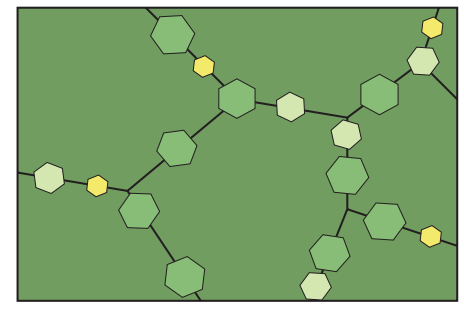

(e) Stage 5

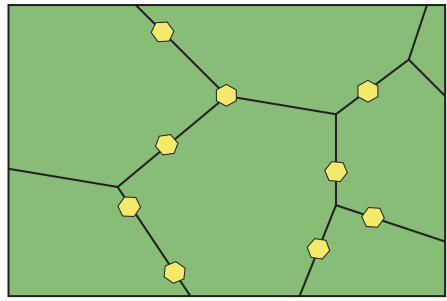

(c) Stage 3

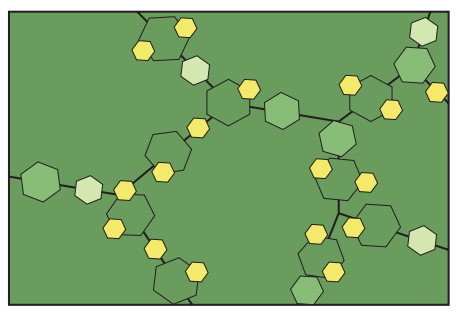

(f) Stage 6

Figure 1: Schematic diagram showing the theory behind the recrystallisation model

The microstructural information shown in Figure 1 must be stored within a model in a form that can be both easy to manipulate and efficient to store and access. If individual grains are grouped into sets of similar grains, grainsets, the microstructural information can be stored in the form of two interlinked tables. The development of the main table representing the series of schematics in Figure 1 is shown in Table 1. For the current example, the initial matrix (of 7 grains) can be be grouped into a grainset with an average diameter of, for example, $12 \mu \mathrm{m}$, and a low dislocation density (represented here by a value of 1). As this is the only grainset in the system, the area fraction occupied by the grainset, A, is 1 (shown in Table 1 (a)). As deformation begins, the dislocation density within Grainset 1 increases (Figure 1 (b) and Table 1 (b)), although no nucleation of recrystallised grains occurs as the critical dislocation density has yet to be reached. By stage 3 (Figure 1 (c) 
and Table 1 (c)), the dislocation density within the grains in Grainset 1 has reached that of the critical dislocation density, and nucleation of new grains has occurred. In the current example, 9 grains, each with a diameter of $1 \mu \mathrm{m}$ have been nucleated, occupying an area fraction of 0.009. The area fraction of Grainset 1 is reduced, therefore, by 0.009 to 0.991. The location of the new grainset, Grainset 2, needs to be stored. This is achieved using the subsidiary table (Table 2), where it can be seen that the full fraction, 1 , of the grains in Grainset 2 are located on the grain boundaries of Grainset 1. As deformation continues through stages 4 and 5 , the procedure is repeated. A number of points should be noted, however; firstly, the new grains are all nucleated on the boundaries of Grainset 1. This is because the critical dislocation density has yet to be reached in the new grains. Secondly, the number of new grains nucleated is decreasing; due to the unoccupied grain boundary area of Grainset 1 being reduced. During stage 6, however, nucleation occurs on both Grainset 1 and 2. This is recorded in the subsidiary table, and it can also be seen that the number of new nuclei formed is much greater than in the previous stages, a result of an increase in grain boundary area available to nucleate on.

\begin{tabular}{ccccc}
\hline \hline $\mathrm{G}$ & $\mathrm{N}$ & $d$ & $\mathrm{~A}$ & $\rho$ \\
\hline 1 & 7 & 12 & 1.000 & 1 \\
\hline \hline
\end{tabular}

(a) Stage 1

\begin{tabular}{ccccc}
\hline \hline $\mathrm{G}$ & $\mathrm{N}$ & $d$ & $\mathrm{~A}$ & $\rho$ \\
\hline 1 & 7 & 12 & 0.959 & 4 \\
2 & 9 & 2 & 0.036 & 3 \\
3 & 5 & 1 & 0.005 & 1 \\
\hline \hline
\end{tabular}

(d) Stage 4

\begin{tabular}{ccccc}
\hline \hline $\mathrm{G}$ & $\mathrm{N}$ & $d$ & $\mathrm{~A}$ & $\rho$ \\
\hline 1 & 7 & 12 & 1.000 & 2 \\
\hline \hline
\end{tabular}

(b) Stage 2

\begin{tabular}{ccccc}
\hline \hline $\mathrm{G}$ & $\mathrm{N}$ & $d$ & $\mathrm{~A}$ & $\rho$ \\
\hline 1 & 7 & 12 & 0.896 & 5 \\
2 & 9 & 3 & 0.080 & 4.5 \\
3 & 5 & 2 & 0.020 & 3 \\
4 & 4 & 1 & 0.004 & 1 \\
\hline \hline
\end{tabular}

(e) Stage 5

\begin{tabular}{ccccc}
\hline \hline $\mathrm{G}$ & $\mathrm{N}$ & $d$ & $\mathrm{~A}$ & $\rho$ \\
\hline 1 & 7 & 12 & 0.991 & 3 \\
2 & 9 & 1 & 0.009 & 1 \\
\hline \hline
\end{tabular}

(c) Stage 3

\begin{tabular}{ccccc}
\hline \hline $\mathrm{G}$ & $\mathrm{N}$ & $d$ & $\mathrm{~A}$ & $\rho$ \\
\hline 1 & 7 & 12 & 0.780 & 6 \\
2 & 9 & 4 & 0.143 & 6 \\
3 & 5 & 3 & 0.045 & 4.5 \\
4 & 4 & 2 & 0.016 & 3 \\
5 & 17 & 1 & 0.017 & 1 \\
\hline \hline
\end{tabular}

(f) Stage 6

Table 1: Main table holding the microstructure information

\begin{tabular}{c|ccccc}
\hline \hline & 1 & 2 & 3 & 4 & 5 \\
\hline 2 & 1 & - & - & - & - \\
3 & 1 & - & - & - & - \\
4 & 1 & - & - & - & - \\
5 & 0.25 & 0.75 & - & - & - \\
\hline \hline
\end{tabular}

Table 2: Subsidiary table

The model is designed to produce graphs of volume fraction recrystallised, but also histograms showing the number of grains present and of volume fraction occupied for each ASTM grain size. Figure 2 shows an overview of the model.

Setting the Initial Conditions

The model is designed to work in tandem with any finite element package, requiring only a temperature and strain rate / strain history, and an initial microstructure as inputs. These histories can be discretised into a number of steps (set by the user), each with a time step, $d t$, temperature, $T$, and increase in strain, $d \varepsilon$. The initial microstructure can be set as a uniform grain size, or input in the form of the tables shown in Tables 1 (f) and 2. 


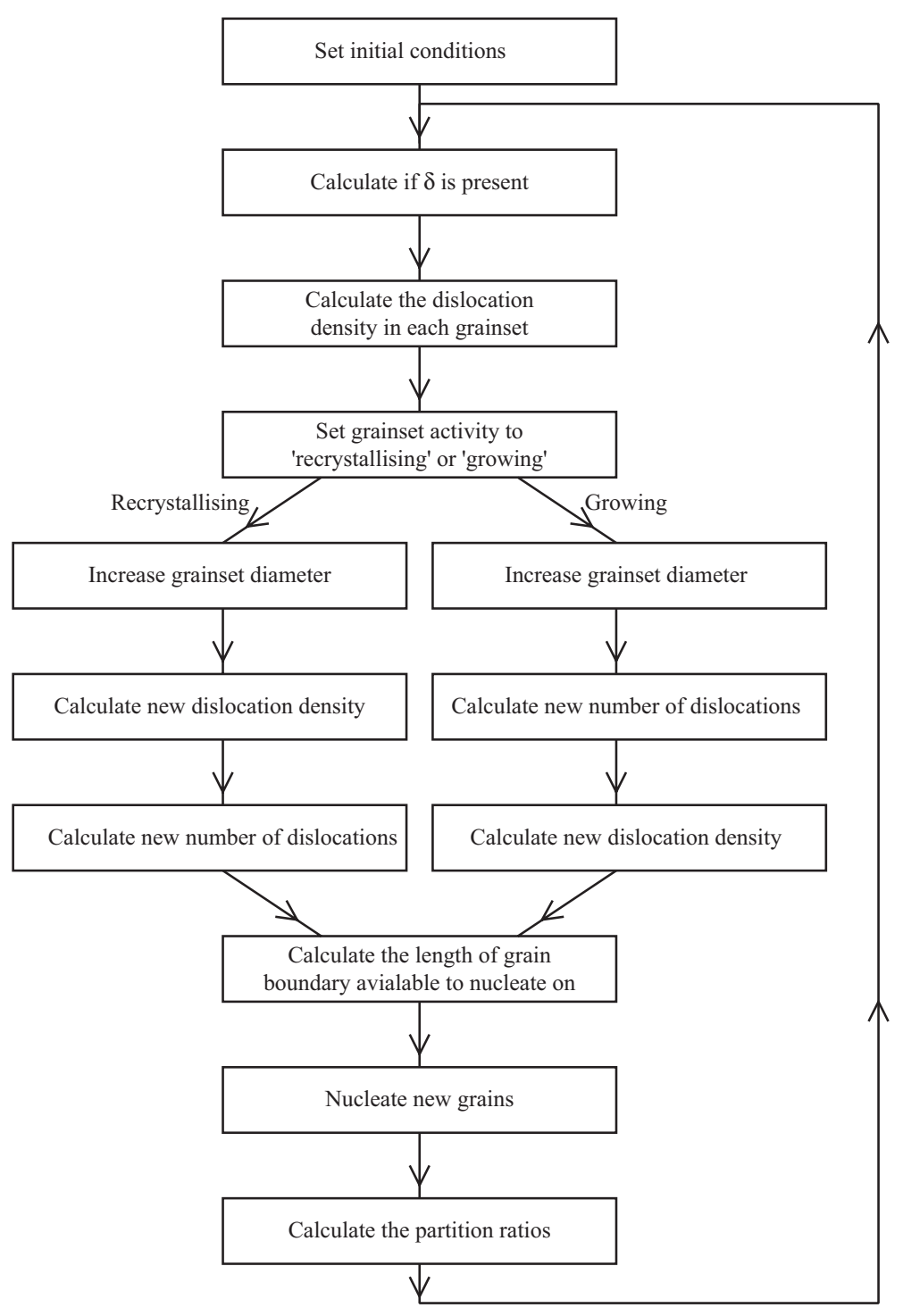

Figure 2: Outline of the model

The use of a list of steps, each having an associated temperature, time step, and increase in strain, results in the model being able to handle dynamic and metadynamic recrystallisation using the same computer code (the increase in strain, $d \varepsilon$, simply being 0 to simulate metadynamic behaviour).

\section{Prediction of $\delta$ Precipitation}

In order to accurately predict both the velocity of moving grain boundaries, and the nucleation rate of recrystallised grains, the presence or lack of precipitates must be accounted for. To predict the onset of $\delta$ precipitation, an existing TTT diagram can be used in conjunction with a Scheil calculation [14-16]. Equation 1 shows the Scheil additive function, where $t$ is the time required to initiate precipitation at a given temperature, and $\Delta t$ is the time spent at that temperature. The model uses the simple criterion that, when the summation equals $1, \delta$ is present, and both the movement of grain boundaries and the nucleation of new grains are inhibited. 


$$
\sum_{i} \frac{\Delta t_{i}}{t_{i}}=1
$$

When the temperature is raised above that of the $\delta$ solvus $\left(1022^{\circ} \mathrm{C}\right)$, and remains above it for more than 5 seconds, any precipitates are assumed to go into solution, and the Scheil additive summation begins again when the temperature falls below that of the solvus.

Calculating the Dislocation Density in Each Grainset

After the presence of $\delta$ has been predicted the new dislocation densities within each grainset need to be calculated. McQueen [17] has noted that dislocation density varies linearly with strain, thus, for this model, an increase in strain, $d \varepsilon$, results in a fixed number of dislocations being added to the system, $d n$ (Equation 2). Although this is an over-simplification, many assumptions and simplifications need to be made when developing a model. The number of dislocations added due to the increase in strain is assumed to be independent of temperature and strain rate.

$$
d n=K d \varepsilon
$$

The value of $K$ has been calculated to be approximately $6 \cdot 6 \times 10^{14}$, calculated using Equation 3, where $\rho_{c}$, the critical dislocation density for the onset of recrystallisation is $1 \times 10^{14} \mathrm{~m}^{-2}[18] ; \rho_{\text {unr } x}$, the dislocation density of a newly recrystallised grain, is $1 \times 10^{12} \mathrm{~m}^{-2}[7]$, and $\varepsilon_{\text {crit }}$ is the observed critical strain (approximately 0.15 in the case of IN 718 [19]). The value of $K$ is, therefore, fitted to experimental data such that, excluding recovery processes and assuming that the original structure has a uniform dislocation density equal to $\rho_{\text {unr }}$, dynamic recrystallisation would begin at the assumed critical strain of 0.15.

$$
K=\frac{\rho_{c}-\rho_{u n r x}}{\varepsilon_{\text {crit }}}
$$

Once the number of dislocation being added to the system in the timestep has been calculated, they must be distributed into the grainsets correctly. Obviously, grainsets occupying a large area will receive more dislocations then those only occupying a small area. The dislocation density of the grainsets also influences the distribution of the dislocations, however, with the grainsets with a low dislocation density receiving more dislocations than already heavily deformed grainsets.

\section{Setting The Activity of Each Grainset}

In order to accurately calculate the manner in which each grainset is going to behave, it is necessary to assign each grainset an activity. These activities can be either recrystallising, impinging, or growing. A recrystallising grainset is one that both has a dislocation density lower than that of the matrix and has space to grow into. An impinging grainset is identified as it still has a dislocation density lower than that of the matrix, but has very little space to grow into. The lack of space within which to grow will be a result of nucleation events having occurred after that of the grainset in question. A grainset designated as growing has, by definition, stopped recrystallising and has the same dislocation density as that matrix and is treated by the model as part of the matrix.

The growth rate of a recrystallising grainset will be calculated as a function of the dislocation density difference between it and the surrounding grains, whereas the velocity of 
grain boundaries belonging to impinging or growing grainsets will be calculated simply as a function of temperature.

It should be noted here that it is probable that the volume fraction of grainsets designated as recrystallising will be significantly lower than the actual volume fraction recrystallised. When a newly recrystallised grainset ceases recrystallising and begins growing, it still counts as a recrystallised area.

Growing the Grains by Recrystallisation

To calculate the growth rate of grains within a grainset, a number of factors need to be taken into account. These factors include the temperature, the magnitude of the driving force causing the boundaries to migrate, and the fraction of the system the grainset has to grow into. Equation 4 shows that the growth rate of a particular grainset, $G$, is calculated by multiplying the maximum possible growth rate for that grainset, $G_{\max }$, by a driving force factor, $D F$, and the area fraction remaining for that grainset to grow into, $A^{\text {rem }}$.

$$
G_{(x)}=G_{\max } \times D F_{(x)} \times A_{(x)}^{r e m}
$$

$G_{\max }$ is a purely kinetic based value, and represents the growth rate of the grainset if, for example, only 1 grain had nucleated and was growing into a matrix with an infinitely high dislocation density and with nothing to impede it's progress. The equation used to calculate the maximum possible growth rate is shown as Equation 5 (where $R$ is the ideal gas constant, and $T$ is the temperature in Kelvin). The value for the activation energy, $\mathrm{Q}$, has been set to $240000 \mathrm{~J} \mathrm{~mol}^{-1}$ [9], and the factor $c_{r x}$ has been fitted to experimental data and set to $3 \cdot 3 \times 10^{10} \mathrm{~mm} \mathrm{~s}^{-1}$.

$$
G_{\text {max }}=c_{r x} \exp \left(\frac{-Q}{R T}\right)
$$

Values for $D F_{(x)}$ and $A_{(x)}^{r e m}$ are calculated using the dislocation density of the grainset in question and that of the surrounding matrix.

Growing Grains by Normal Grain Growth

The growth rate for unrecrystallised grains has been calculated by fitting to the equation provided by Huang et al. [5] (Equation 6).

$$
d=\sqrt{d_{0}^{2}+9 \cdot 44 \times 10^{19} t \exp \left(\frac{-467114 \cdot 7}{R T}\right)}
$$

Calculating the Length of Grain Boundary Available to Nucleate On

Similar to the model proposed by Ding and co-workers [20], the criteria for the onset of dynamic recrystallisation is a critical dislocation density rather than a critical strain. The critical dislocation density used in this model is $1 \times 10^{14} \mathrm{~m}^{-2}$, a value obtained by Sandström and Lagneborg [18] for pure nickel. Although it has been proposed that the critical dislocation density is a function of temperature and strain rate [18], this model assumes it is constant.

The length of grain boundary available for nucleation is calculated using both the main table, and the subsidiary table. Initially, a list is made of those grainsets with a dislocation density higher than that of the critical dislocation density. This list is then shortened to 
include only those grainsets that are not currently recrystallising. Each of these grainsets is then analysed and the available boundary length calculated using Equation 7 where $P_{(x)}$ is the total perimeter of the grainset being analysed, $x, N_{(y)}$ and $d_{(y)}$ are the number of grains and diameter of the grains in another grainset, $y$, and $F r_{(x, y)}$ represents the fraction of Grainset $y$ located on Grainset $x$.

$$
L_{(x)}=P_{(x)}-\sum_{y=x+1}^{n}\left(N_{(y)} d_{(y)} F r_{(x, y)}\right)
$$

\section{Nucleating New Grains}

As has already been discussed, the nucleation of new grains is only permitted on stationary grain boundaries, an assumption made as it is unfavourable for a nucleation event to occur on the boundary of a currently recrystallising grain.

For the purposes of this model, the diameter of a newly nucleated recrystallised grain is fixed at $0.25 \mu \mathrm{m}$. Bailey [21] and Humphreys [22] have stated that the theoretical critical radius for a new recrystallised grain should be $0.5 \mu \mathrm{m}$, yet TEM observations of IN 718 deformed at $980^{\circ} \mathrm{C}$ have shown dynamically recrystallised grains with diameters of approximately $350 \mathrm{~nm}$ (Figure 3). It is reasonable to assume that the grains in the image have grown to a certain extent, and that the original size was no greater than $250 \mathrm{~nm}$.

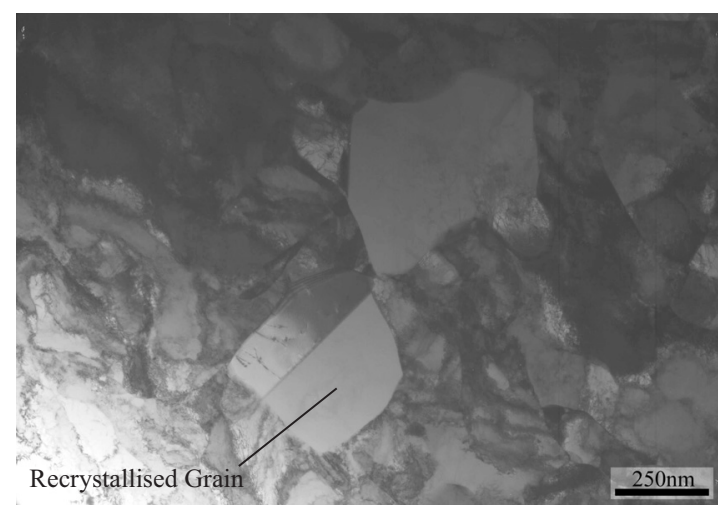

Figure 3: TEM micrograph showing recrystallised grains in IN 718

When a new grainset is added, the dislocation density is set to $1 \times 10^{12} \mathrm{~m}^{-2}$, a value identified by Marty et al. [7] as being the lowest dislocation density observed in polycrystals.

$\underline{\text { Increasing the Time Step }}$

Once the nucleation of new grains has been calculated, the information for each grainset in the system can be updated, and the values in both the main and subsidiary tables are refreshed. The model can now move onto the next step and repeat the procedure on the new microstructure.

\section{Comparison with Industrial Forgings}

The model has been validated against a typical IN 718 forging produced by Firth Rixson Forgings Ltd. The process route consists of an initial 3 hour subsolvus furnace soak, a 
preforming operation, a 1 hour subsolvus furnace reheat, and a final forging operation before being left to cool in air. Figure 4 shows the shape of the workpiece and of the dies during each stage.

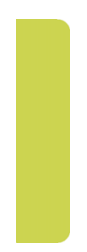

(a) Initial billet

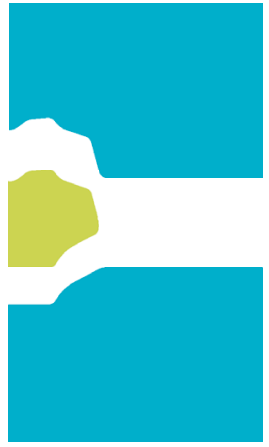

(b) Preform stage

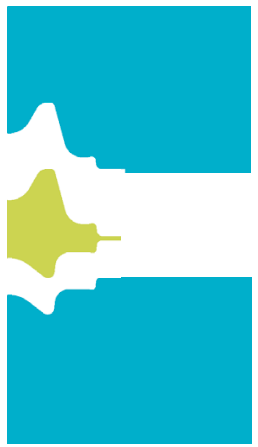

(c) Final stage

Figure 4: Diagrams of the workpiece and dies for the stages of the forging process carried out at Firth Rixson Ltd

In order to validate the model, the microstructure within the workpiece had to be tracked during the forging process. This was accomplished by taking samples from the original billet both before and after the initial furnace soak period, after the preforming operation, after the reheating stage, and after the final operation. Figure 5 shows the temperature, strain, and strain rate experienced by the top, centre, and bottom of the workpiece during the process route. The temperature has been normalised to that of the furnace temperature.

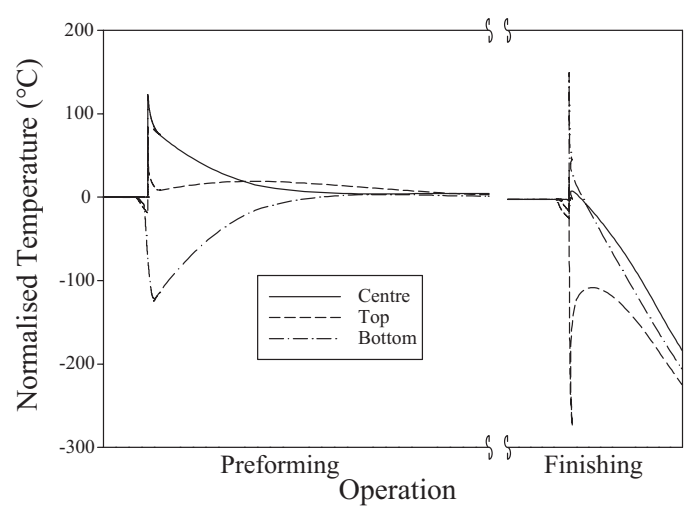

(a) Temperature

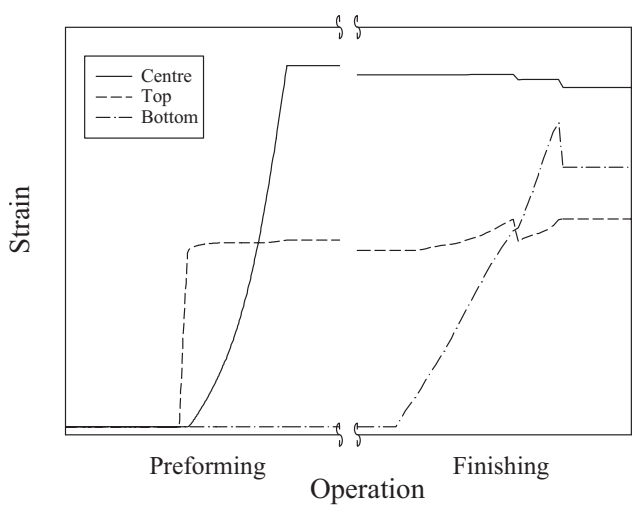

(b) Strain

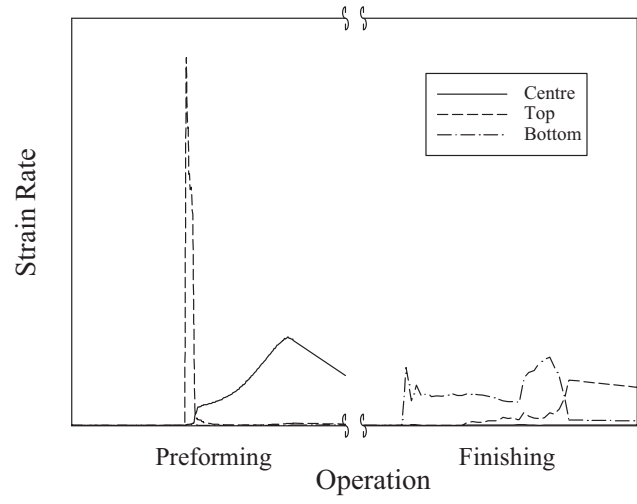

(c) Strain Rate

Figure 5: Diagrams of the workpiece and dies for the stages of the forging process carried out at Firth Rixson Ltd 
Figures 6 (a), (d), and (g) show the microstructure after the preforming operation at the top, centre, and bottom of the workpiece respectively. The microstructures observed can be easily related to the temperature and strain profiles shown in Figure 5.

The micrograph showing the structure at the top of the workpiece after the preforming operation (Figure 6 (a)) shows that this area has undergone recrystallisation, as it has experienced a strain greater than that of the critical strain. As the temperature in this region has remained cool during deformation, and has cooled down rapidly, the recrystallised grains have not grown substantially, resulting in an average grain diameter of approximately $20 \mu \mathrm{m}$ (ASTM 8). The model accurately predicts this, showing a grain size distribution centred around ASTM 8.5 (Figure $6(\mathrm{~b})$ ) and indicates that $\delta$ precipitates are still present in the structure (Figure $6(\mathrm{c})$ ).

The centre of the preform is subjected to a large amount of strain and the resulting adiabatic heating increases the temperature by more that $100^{\circ} \mathrm{C}$. This causes rapid progression of dynamic recrystallisation and the solutioning of the $\delta$ precipitates. The temperature remains high enough for a sufficient period to allow full recrystallisation to occur and for the grains to grow to an average diameter of approximately $45 \mu \mathrm{m}$ (ASTM 6), shown in Figure 6 (d). The predicted microstructure after the preforming operation and cool-down is an average grain size of approximately $37 \mu \mathrm{m}(\approx \mathrm{ASTM} 6.5)$ without any precipitates present (Figures 6 (e) and (f)).

The image showing the bottom of the billet after the preforming operation (Figure $6(\mathrm{~g})$ ) shows no recrystallisation as this is in a dead zone within the preform where the strain is almost zero. During the heat-up, deformation, and cool down period, the grain size in the dead zone has grown to approximately $55 \mu \mathrm{m}$ (ASTM 5.5). Once again, this microstructure is accurately predicted by the model, shown by Figures $6(\mathrm{~h})$ and (i).

Final Operation

The progression of the microstructures from those seen in the preform to those observed in the final forging can also be explained using the thermomechanical histories of the three points during the final forging operation (Figure 5).

The top of the workpiece experiences sufficient strain to initiate the onset of recrystallisation, but due to die chill, the new grains do not have time to grow to any great extent. The structure is, therefore, refined from that after the preforming operation (Figure 6 (a)) to that seen in Figure 7 (a) containing the initial $\delta$ precipitates formed during the initial 3 hour soak period, and newly recrystallised grains of approximately $12 \mu \mathrm{m}$. Figure 7 (c) describes the grain refinement during the two operations. A greater refinement is observed during the final forging operation for two reasons. Firstly, the initial grain size is smaller, resulting in more grain boundary area for new grains to nucleate on, and secondly, because the workpiece is left to cool rather than being put into a furnace at $983^{\circ} \mathrm{C}$. The predicted final microstructure is a grain size of approximately $8 \mu \mathrm{m}(\approx \mathrm{ASTM} 10 \cdot 5)$ with $\delta$ precipitates present, almost exactly the microstructure seen in the final workpiece.

The centre of the workpiece has remained in the same state during the final forging operation. This region experiences no strain during this operation, and small amounts of $\delta$ are present on the grain boundaries to slow grain growth (Figure $7(\mathrm{~d})$ ). The model predicts that the grains continue to grow until $\delta$ phase is precipitated on the boundaries (Figure 7 (f)). The grain growth predicted here is, however, much greater than is observed in the real forging, where almost no difference in grain size is observed between the cooled 

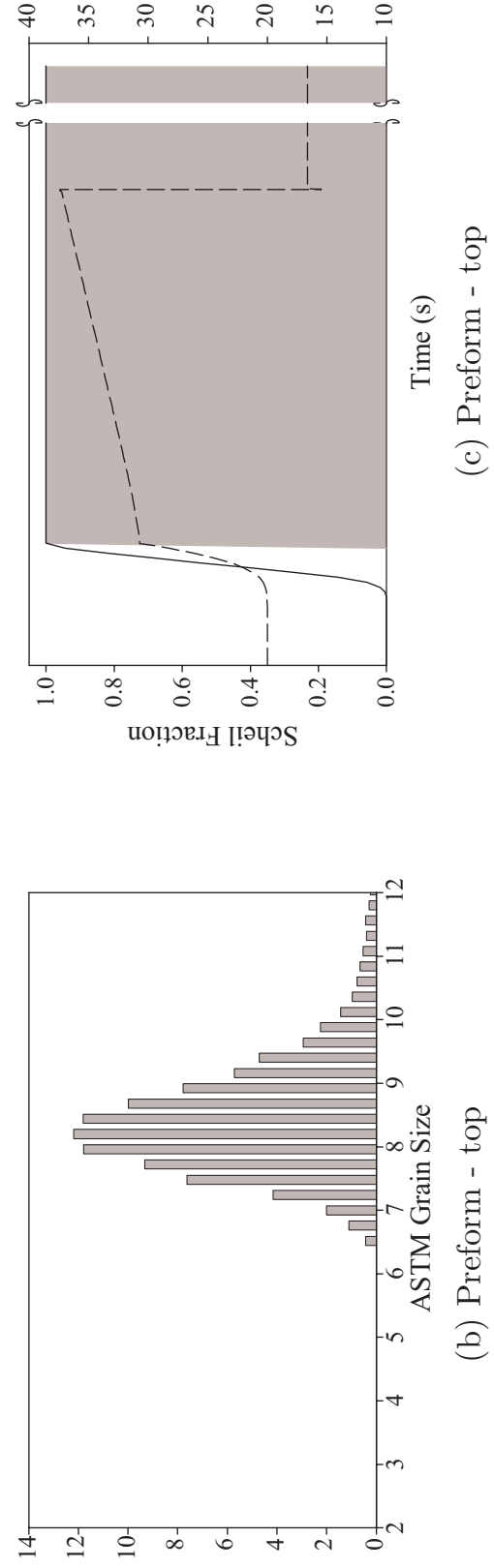

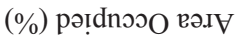

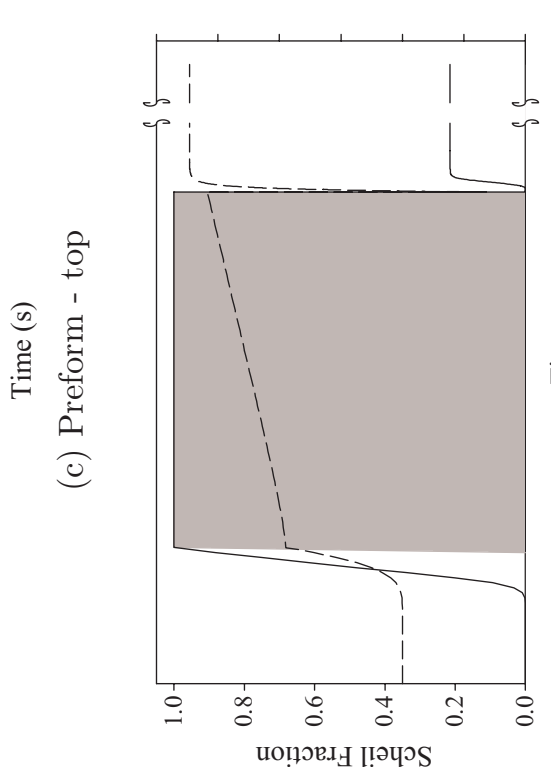

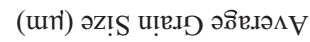

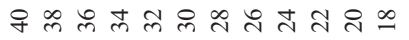

클

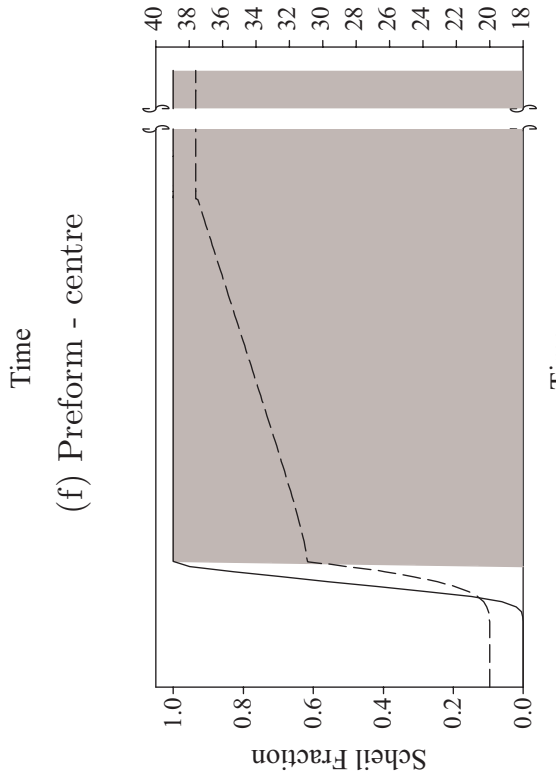

$\underset{7}{7}$

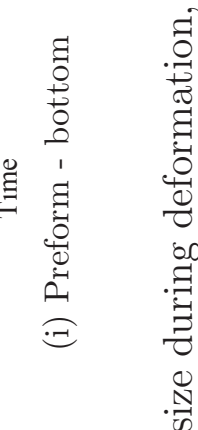

.

\begin{tabular}{l}
8 \\
80 \\
0 \\
0 \\
0 \\
0 \\
0 \\
0 \\
\hline
\end{tabular}
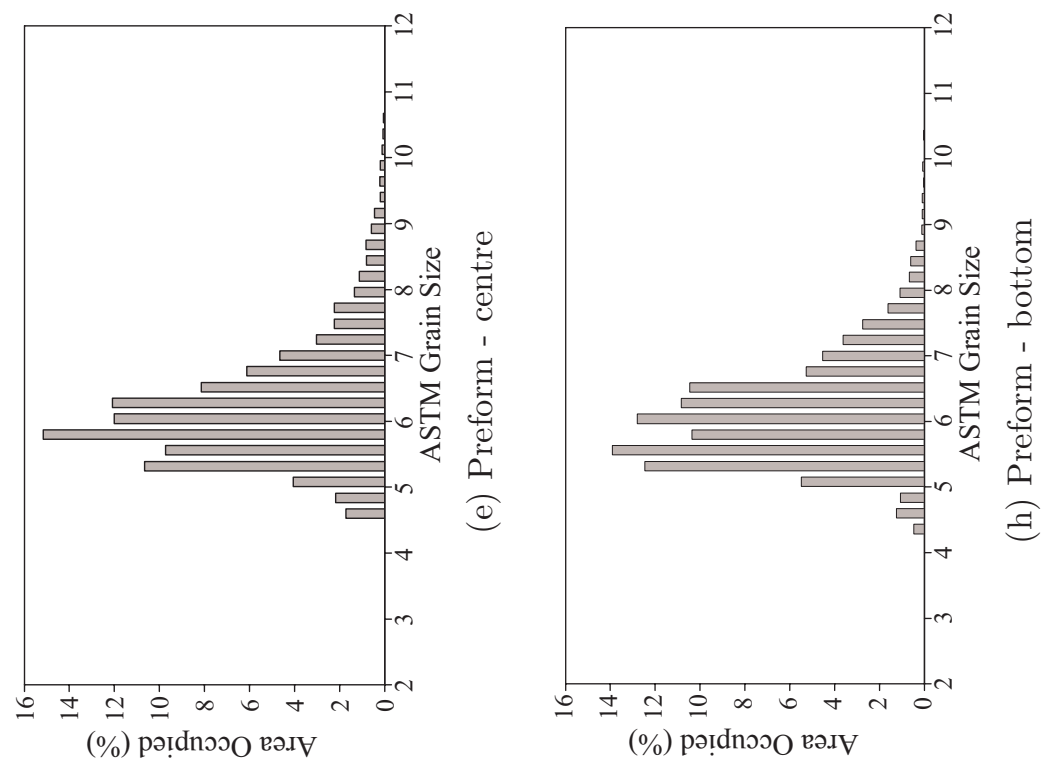

30

完.

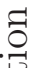

प्र

दू ०

bo

.

ฮี

है

तु

要焉

.

.$\exists \stackrel{1}{2}$
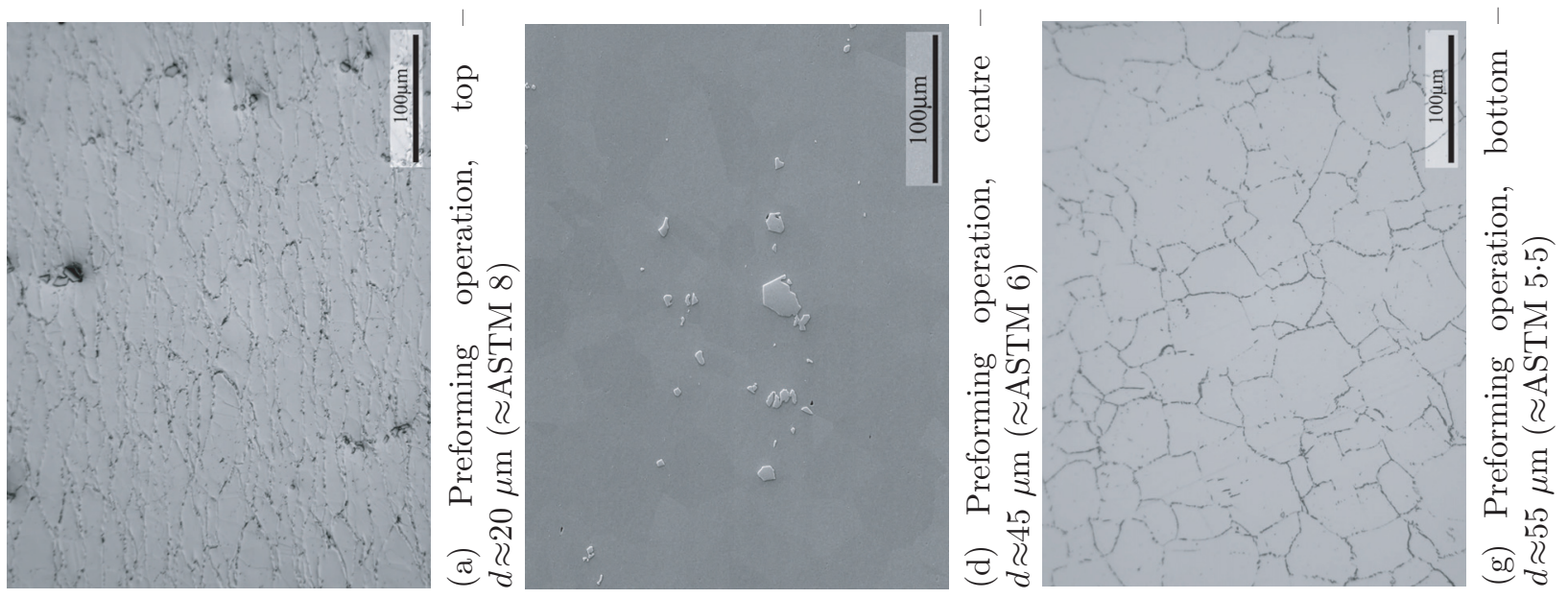


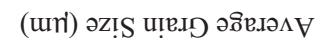
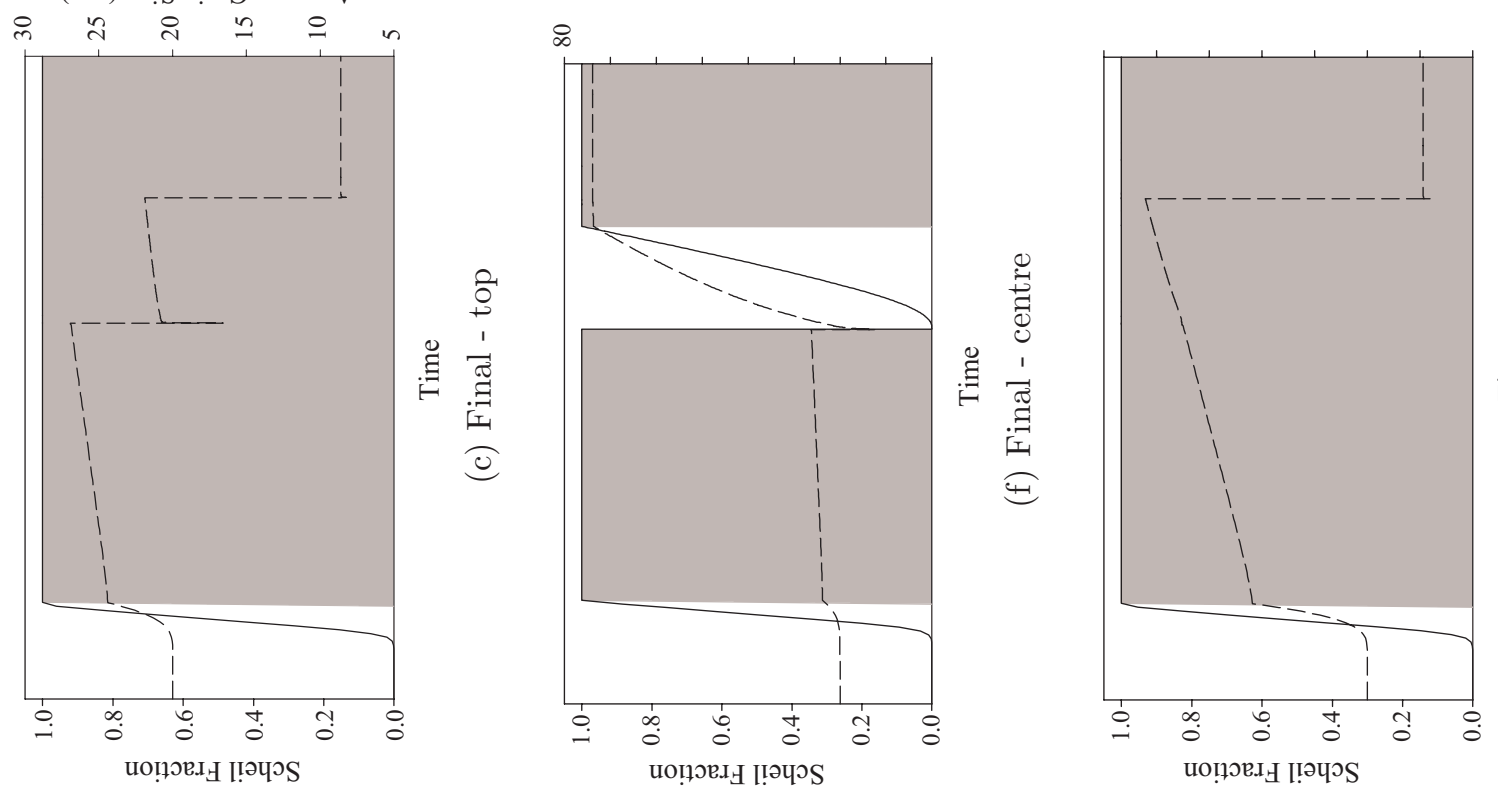

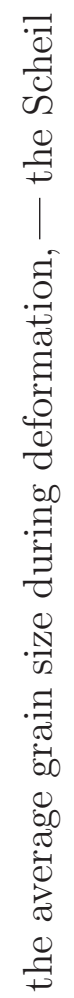

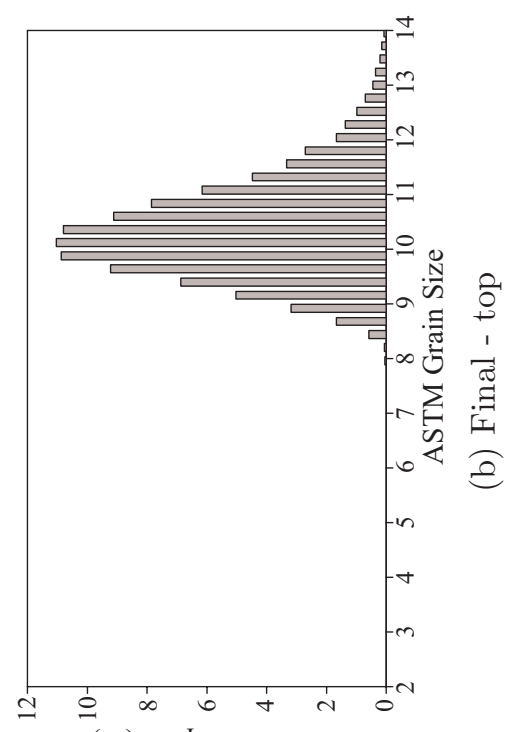

(\%) pəİdnooO вә.I

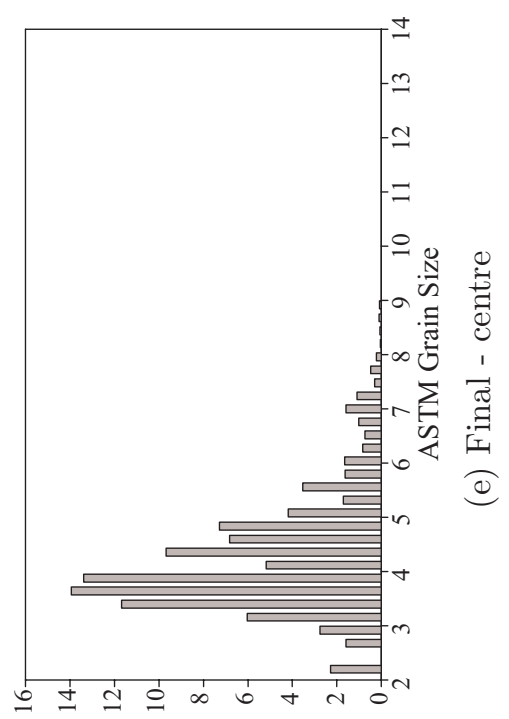

(\%) pəİdnoo в вәIV

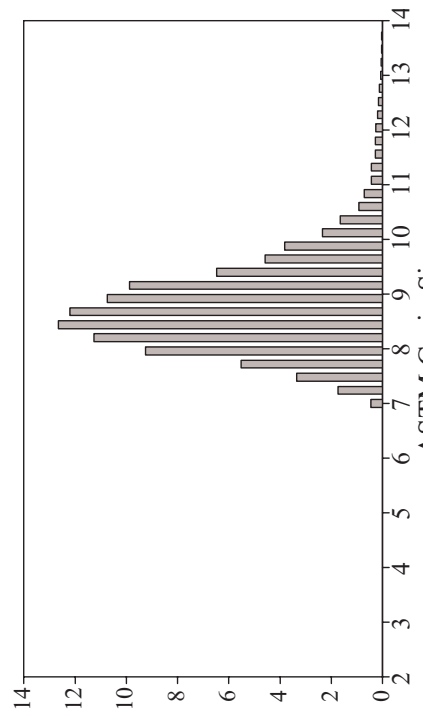

(\%) pə⿱亠巾中nэวO вә.IV
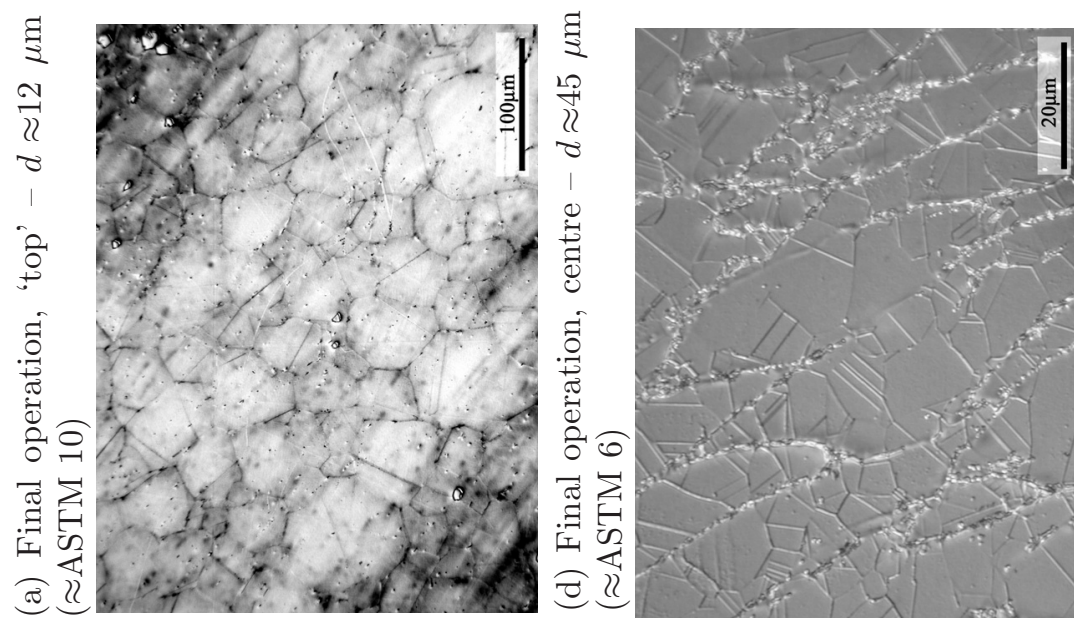

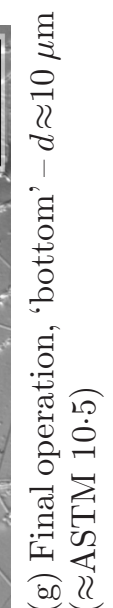

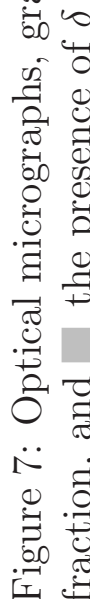


preform and final workpiece. The final grain size predicted by the model is approximately $75 \mu \mathrm{m}(\approx \mathrm{ASTM} 4)$, whereas the observed grain size is approximately $45 \mu \mathrm{m}(\approx \mathrm{ASTM} 6)$. The excessive grain growth predicted by the model can be attributed to the relationship between the velocity of the migrating grain boundaries and the precipitation of $\delta$. It is likely that the time between the preforming forging operation and the onset of $\delta$ precipitation is less than the time predicted by the model. As previously explained, the model uses an empirically derived TTT diagram and a Scheil additive calculation to predict the onset of $\delta$ precipitation. This TTT diagram will have been produced with the niobium evenly distributed throughout the matrix. As the rate of nucleation and growth of $\delta$ phase in IN 718 is diffusion controlled, the time before the onset of precipitation using this TTT diagram will be much greater than if the niobium was clustered in certain areas. This is, however, the case in the workpiece immediately after the preforming operation. When the temperature rises above the $\delta$ solvus during the preforming operation, the niobium will not instantly redistribute itself uniformly within the matrix. Therefore, when the temperature falls below that of the solvus, the onset of precipitation of $\delta$ will occur much faster than that predicted by the model, resulting in a finer final grain size.

The bottom of the workpiece is subjected to a large strain during the final forging operation, thus causing recrystallisation to occur (as can be seen in Figure 7 (g)). As the $\delta$ precipitates are still present from the initial soak period, the deformed nature of the original grains can clearly be seen, with newly formed grains visible within the older structure. Although the temperature at the bottom of the workpiece does increase above that of the $\delta$ solvus during this last operation, the precipitates do not return into solution as the time the area remains above the solvus is not sufficiently long enough. The predicted microstructure for the bottom of the workpiece (Figures 7 (h) and 7 (i)) shows good agreement to that seen experimentally. After the initial precipitation of $\delta$ during the first soak stage, the precipitates remain throughout the forging procedure. As the $\delta$ phase is present, the growth of these precipitates if inhibited, resulting in a final predicted grain size of approximately $14 \mu \mathrm{m}(\approx \mathrm{ASTM} 9)$, similar to that observed $(10 \mu \mathrm{m}$ (ASTM 10.5)).

\section{Conclusions}

A model has been developed that, using the thermomechanical history produced by a finite element software package, can accurately predict the microstructural evolution of Inconel 718. The new model has been shown to predict both the grain size and presence of $\delta$ precipitates throughout the production of a typical two-stage forging procedure. Only a small number of constants need to be altered for the model to predict the microstructural evolution of other Ni-base superalloys.

\section{References}

[1] B. Antolovich and M. Evans. Predicting grain size evolution of UDIMET ${ }^{\circledR}$ alloy 718 during the "cogging" process through the use of numerical analysis. Superalloys 2000, pages 39-47, 2000.

[2] A. J. Brand, K. Karhausen, and R. Kopp. Microstructural simulation of nickel base alloy inconel 718 in production of turbine discs. Materials Science and Technology, 12:963-969, November 1996.

[3] D. E. Camus, R. A. Jaramillo, J. A. Plyburn, and F. S. Suarez. Evolution of microstructure during hot rolling of inconel alloys 625 and 718. Superalloys 718, 625, 706 and Various Derivatives, pages 291-302, 1997. 
[4] C. A. Dandre, S. M. Roberts, R. W. Evans, and R. C. Reed. Microstructural evolution of inconel 718 during ingot breakdown: Process modelling and validation. Materials Science and Technology, 16(1):14-25, January 2000.

[5] D. Huang, W. T. Wu, D. Lambert, and S. L. Semiatin. Computer simulation of microstructure evolution during hot forging of waspaloy and nickel alloy 718. In R. Srinivasan, editor, Microstructure Modeling and Prediction During Thermomechanical Processing, pages 137-146. TMS, November 2001.

[6] R. A. Jaramillo, F. S. Suarez, J. A. Plyburn, D. E. Camus, and R. W. Evans. Evaluation of an inconel ${ }^{\circledR}$ alloy 718 microstructural evolution model. Superalloys 718, 625, 706 and Various Derivatives, pages 257-266, 1997.

[7] B. Marty, J. Y. Guedou, P. Gergaud, and J. L. Lebrun. Recrystallisation and work-hardening prediction during forging process of inconel 718. Superalloys 718, 625, 706 and Various Derivatives, pages 331-342, 1997.

[8] M. C. Mataya, E. E. Nilsson, and G. Krauss. Comparison of single and multiple pass compression tests used to simulate microstructural evolution during hot working of alloys 718 and 304l. Superalloys 718, 625, 706 and Various Derivatives, pages 331-343, 1994.

[9] S. C. Medeiros, Y. V. R. K. Prasad, W. G. Frazier, and R. Srinivasan. Microstructural modeling of metadynamic recrystallization in hot working of IN 718 superalloy. Materials Science and Engineering A, 293:198-207, 2000.

[10] N. Srinivasan and Y. V. R. K. Prasad. Microstructural control in hot working of IN-718 superalloy using processing map. Metallurgical and Materials Transactions A, 25:2275-2284, October 1994.

[11] J. M. Zhang, Z. Y. Gao, J. Y. Zhuang, and Z. Y. Zhong. Mathematical modelling of the hot-deformation behaviour of superalloy IN718. Metallurgical and Materials Transactions A, 30:2701-2712, October 1999.

[12] L. X. Zhou and T. N. Baker. Effects of strain rate and temperature on deformation behaviour on IN718 during high temperature deformation. Materials Science and Engineering A, 177:1-9, 1994.

[13] H. J. McQueen and J. J. Jonas. Recovery and Recrystallization During High Temperature Deformation, volume 6 of Treatise on Materials Science and Technology, pages 393-493. Academic Press, 1975.

[14] I. I. Boyadjiev, P. F. Thomson, and Y. C. Lam. Computation of the diffusional transformation of continuously cooled austenite for predicting the coefficient of thermal expansion in the numerical analysis of thermal stress. ISIJ International, 36(11):1413-1419, 1996.

[15] H. K. D. H. Bhadeshia. Thermodynamic analysis of isothermal transformation diagrams. Metal Science, 16:159-165, March 1982.

[16] Y. T. Zhu, T. C. Lowe, and R. J. Asaro. Assessment of the theoretical basis of the rule of additivity for the nucleation incubation time during continuous cooling. Journal of Applied Physics, 82(3):1129-1137, August 1997.

[17] H. J. McQueen. The experimental roots of thermomechanical treatments for aluminium alloys. Journal of Metals, 32:17-26, February 1980.

[18] R. Sandström and R. Lagneborg. A model for hot working occuring by recrystallization. Acta Metallurgica, 23:387-398, March 1975.

[19] R. P. Guest and S. Tin. The effect of $\delta$ precipitates on the recrystallisation behaviour of IN 718. In 6 th International Charles Parsons Turbine Conference, pages 703-719, 16th-18th September 2003.

[20] R. Ding, Z. X. Guo, W. Sha, and A. Wilson. Microstructural characterisation and modelling of a ti-6al-4v alloy during thermomechanical processing in the $\beta$ phase field. In M. R. Winstone, editor, Titanium Alloys at Elevated Temperature: Structural Development and Service Behaviour, pages 29-39. IOM Communications Ltd, 2001.

[21] J. E. Bailey. Electron microscope observations on the annealing processes occurring in cold-worked silver. Philosophical Magazine, 5:833-842, 1960.

[22] F. J. Humphreys. The nucleation of recrystallization at second phase particles in deformed aluminium. Acta Metallurgica, 25:1323-1344, 1977. 